\title{
HVAC early fault detection using a fuzzy logic based approach
}

\author{
Victor Martinez-Viol $^{1}$, Eva M. Urbano ${ }^{1}$, Miguel Delgado-Prieto ${ }^{1}$ and Luis Romeral ${ }^{1}$ \\ ${ }^{1}$ MCIA Research Center, Department of Electronical Engineering \\ Universitat Politècnica de Catalunya \\ Rambla Sant Nebridi, 22, 08222 Terrassa (Spain) \\ Phone/Fax number:+0034 9373985 18, e-mail: victor.martinez.viol@upc.edu, eva.maria.urbano@upc.edu, \\ miguel.delgado@upc.edu, luis.romeral@upc.edu
}

\begin{abstract}
The need for improving the energy efficiency of existing buildings has driven to the implementation of building energy management systems (BEMS) that can help facilities manager to discover and identify problems that may cause energy wastage or affect to occupants' comfort. Modern data-driven fault detection and diagnosis (FDD) make use of the data collected by the building BEMS to provide high accuracy in the revelation of heating, ventilation and air-conditioning (HVAC) system faults. However, these methods need a large amount of faulty data samples during the training, which is an uncommon situation in the real world. The main focus of this paper is to present a methodology to detect faults when the number of faulty samples is low. For this purpose, a regression-based methodology based on an adaptative neuro-fuzzy inference system (ANFIS) chiller model is developed using the data collected from a real use case. The model presents good results, that can be used for benchmarking the machine operation and detect the abnormal operation states.
\end{abstract}

Key words. Fault detection and diagnosis, chiller, adaptative neuro-fuzzy inference system, data-driven.

\section{Introduction}

Residential and commercial buildings consume more than the $30 \%$ of the total energy delivered and this energy is primarily used for heating, ventilation and air-conditioning (HVAC) [1]. The high levels of comfort and indoor air quality combined with the increasing complexity of building services and the legal requirements regarding energy efficiency (e.g. the Energy Performance of Buildings Directive [2]) are increasing the efforts to improve the latter. Among the different techniques explored, fault detection and diagnosis (FDD) systems are demonstrated to be one of the most effective tools to increase building energy efficiency due to the fact that a significant portion of the energy used by HVAC equipment is due to inefficiencies.

Developing an effective FDD method can reduce energy consumption by $15-30 \%$ [3]. Common HVAC faults may remain undetected if they do not have an effect on the room temperature or occupants' comfort. These faults are only noticed when the regular maintenance operations are carried out and by the time the maintenance occurs, HVAC equipment has been constantly deteriorating.
Over the last few years, building energy management systems (BEMS) are being widely implemented as a way of modernizing older buildings. These IT-based solutions monitor building appliances in real-time, streaming high volumes of data which can support the energy performance diagnosis. In general, BEMS can store up to millions of data points but in practice, only a small portion of them are used. Typical energy performance diagnosis is based on supplying graphics such as the energy usage or the room temperature, that can be useful to detect some anomalous behaviours but require an expert to interpret the graphics. Despite the potential of automated FDD systems, they are not being widely used because they are complex and time-consuming as they have to be customized to each building they are applied. In addition, they usually need large amounts of faulty data to properly characterize the system behaviour, which is not the usual situation in realworld scenarios.

\section{A. Related works}

Fault detection methodologies in building HVAC systems can be classified into two subcategories: knowledgedriven and data-driven, also called quantitative or whitebox models and qualitative or black-box models respectively. Knowledge-driven models apply mathematical and physical models whether data-driven models are based on analysing the prior information to extract patterns in the measurements of selected variables without having any physical meaning. Data-driven methods usually require high amounts of historical information and here is where the high volume of data obtained by BEMS become important.

Zhao et al. [4] classify the data-driven FDD methods in classification-based, unsupervised learning-based and regression-based. The first group is based on identifying whether the monitoring data belongs to the normal class or not, being also able to classify the faulty data in several fault classes. This kind of work was carried by Yan et al [5], combining an SVM-based chiller FDD model with a prior feature selection algorithm. Unsupervised learningbased methods have the principal component analysis (PCA) as the most popular algorithm for building energy FDD [6], [7]. It transforms the set of correlated variables 
into a new group of variables which are uncorrelated to each other. It classifies the monitored space into two orthogonal subspaces, which represent normal condition and faulty condition respectively. Finally, regression-based models have as their output a continuous variable, such as the energy consumption or temperature, which is compared with the measured one to identify when the latter is out of the normal range. This kind of models is gaining popularity due to the fact that they can characterize the system only by using normal behaviour data. However, they need a huge amount of data to properly tune their parameters. As stated in the review conducted by Zhao et al. [4] with several examples, the typical approaches in the regression-based models are artificial neural networks (ANN) and support vector regressions (SVR).

In this study, an FDD method is proposed with the objective of using the minimum quantity of faulty data to detect the faults as early as possible in the HVAC equipment. A regression-based model has been developed using an adaptative neuro-fuzzy inference system (ANFIS) to obtain a benchmarking chiller model. ANFIS was first implemented by Jang in 1993 [8] from the combination of a fuzzy logic system, which needs an expert to define the membership functions and the rules of the system, with a neural network used as a learning technique to minimize the membership functions output errors. ANFIS can perform a linear mapping between a set of inputs or features and the desired output with high accuracy like ANNs and, in addition, it has high flexibility on adapting to irregular patterns like the ones present in the equipment energy consumption.

The rest of the article is structured as follows: Section 2 presents an overview of the main steps on the development of the ANFIS model and the FDD scheme, as well as a description of the data used for the study. Section 3 describes the results obtained from applying it to a case study. Finally, in Section 4, the contributions obtained from this paper are highlighted and the concluding remarks are summarized.

\section{Methodology}

The framework proposed aims to detect and diagnose HVAC faults feeding as less as possible faulty samples to the model during its training stage. The flowchart for the proposed algorithm is shown in Fig. 1. First, the raw historical data collected from the plant is pre-processed, including its normalization and a feature selection stage based on genetic algorithms (GA). By performing the latter, the original dataset is reduced to a subset containing fewer variables in order to increase the training efficiency and model accuracy. The data is then divided into training and testing datasets and after that, the ANFIS model is trained. Once the training has finished, the model performance is evaluated using the testing dataset. When performing the online FDD, the raw data coming from the monitoring sensors is pre-processed and fed to the model to then compare its output with the monitored data.

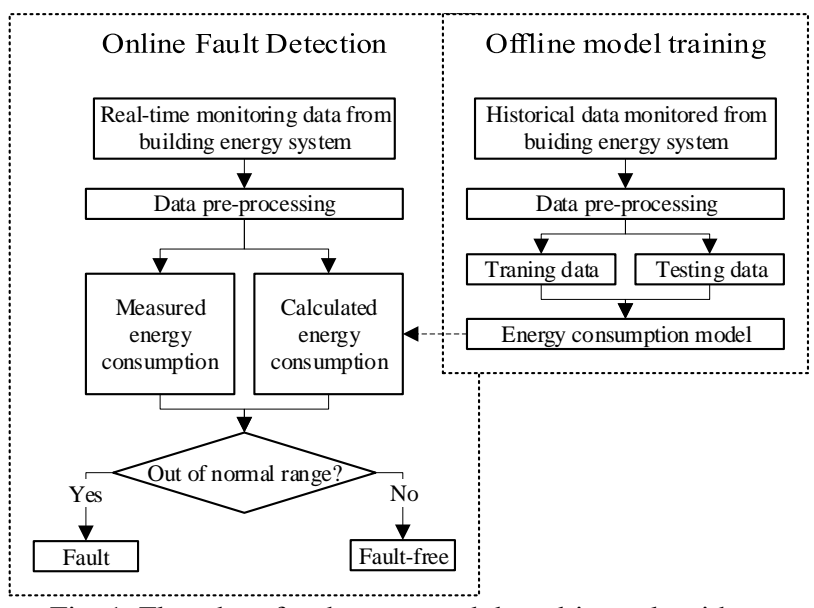

Fig. 1. Flowchart for the proposed data-driven algorithm.

\section{A. Data description}

The model is trained and validated using data collected from the BEMS of a real 3-floor university campus building located in Terrassa (Spain) with a surface of around $2400 \mathrm{~m}^{2}$. The building is a research ecosystem of the Universitat Politècnica de Catalunya which consists of offices and laboratories intended to accommodate several research groups. The installation consists of two electric chillers, two electric heat pumps, one gas boiler and two air handling units (AHU), which manage the energy production, energy distribution, pre-conditioning and airrenewal for the building's spaces. Fig. 2 depicts a diagram of the equipment, which is located on the upper part of the building, on its deck. Both cooling and heating devices have individual electric power meters to measure their electrical consumption, thermal power meters to measure the produced thermal energy.

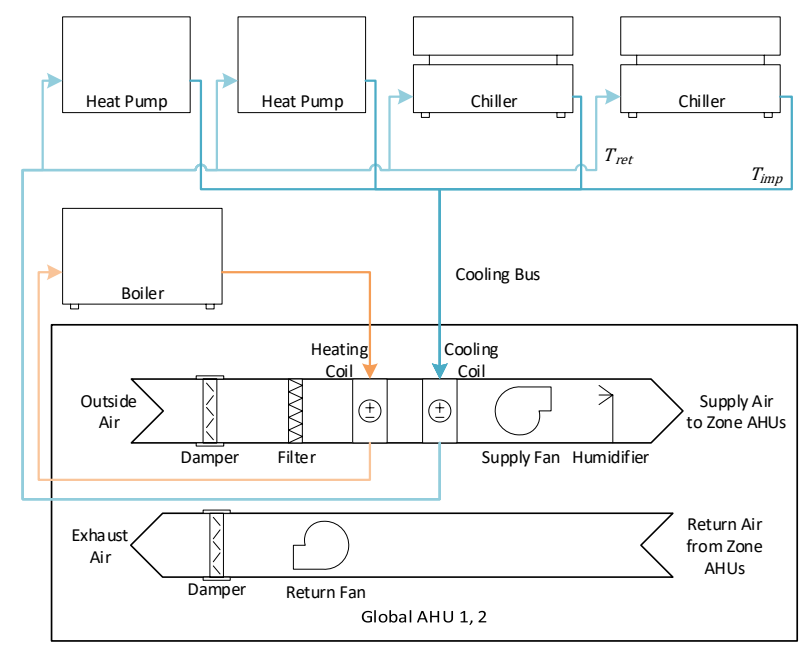

Fig. 2. HVAC plant installed on the case-study building.

Since the chiller is the primary energy consuming source of an HVAC installation, the framework developed has been evaluated on the electrical chillers of the installation. The available data from their operation comprises the periods of spring and summer for the years 2017 to 2018 , with a sampling rate of $15 \mathrm{~min}$. The variables used for the model are listed in Table I. 
Table I. - Variables used to construct the ANFIS FDD model.

\begin{tabular}{|l|l|}
\hline VARIABLE & DESCRIPTION \\
\hline $\boldsymbol{T}_{\text {ext }}$ & Outdoor temperature. \\
\hline $\boldsymbol{T}_{\boldsymbol{i m p}}$ & Chiller impulsion temperature. \\
\hline $\boldsymbol{T}_{\boldsymbol{r e t}}$ & Chiller return temperature. \\
\hline $\boldsymbol{Q}$ & Chiller flow. \\
\hline $\boldsymbol{P}_{\boldsymbol{i n}}$ & Chiller electrical power consumption. \\
\hline $\boldsymbol{P}_{\boldsymbol{\text { out }}}$ & Chiller thermal power. \\
\hline $\boldsymbol{C}_{\boldsymbol{i}}$ & Compressor control variable $(\boldsymbol{i}=\mathbf{1 , 2})$ \\
\hline $\boldsymbol{t}_{\boldsymbol{w}}$ & Day of the week. \\
\hline $\boldsymbol{t}_{\boldsymbol{m i n}}$ & Minute of the day. \\
\hline
\end{tabular}

The chillers in the building have two compressors, therefore if the cooling demand is not too high, only one of the compressors is turned on. This behaviour is shown in Fig. 3. The change is so abrupt that it cannot be modelled properly without the compressor control signals, which are booleans that take 1 as their value when the compressor is enabled.

In order to acquire a dataset including all the required signals from the building, a desktop application was implemented to periodically scan an OPC server linked to the building SCADA system and store the signals in a dedicated time-series database.
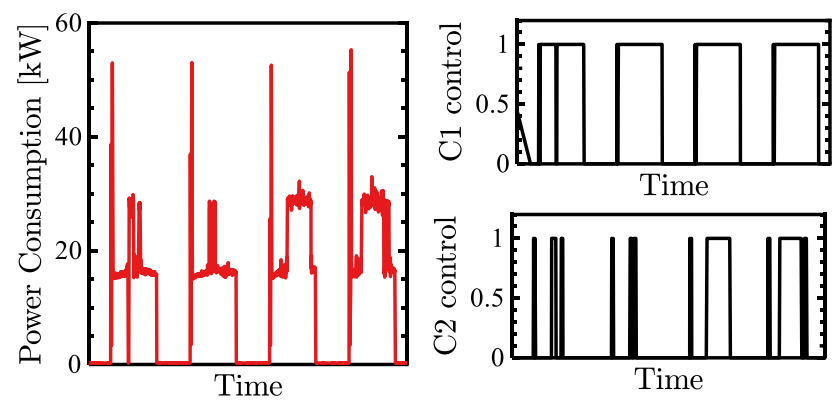

Fig. 3. Chiller consumption abrupt peaks and compressor control signals.

\section{B. Data Normalization}

Given the fact that the magnitude of the variables used is different (e.g. 0 to $3124 \mathrm{~m}^{3} / \mathrm{h}$ for the chiller flow and 10.8 to $35.6{ }^{\circ} \mathrm{C}$ for the external temperature), the accuracy of the prediction will be affected if a normalization step is not applied. According to the past research in the field of energy consumption prognosis, the most common approaches are to express the variables within the range of $[0,1][9]$ or to make the variables have zero mean and unit variance while retaining the shape properties of the original data set [10], [11]. The latter is called $\mathrm{z}$-score normalization and it was the preferred in this study. The function used to apply the normalization can be expressed as:

$$
\phi^{\prime}=\frac{\phi-\mu_{\phi}}{\sigma_{\phi}}
$$

Where $\phi$ and $\phi^{\prime}$ are the value of the variable prior and after the normalization, $\mu_{\phi}$ is the mean value of the variable and $\sigma_{\phi}$ is its standard deviation. After the prediction has been completed, the original value must be restored by multiplying the standardized value by the standard deviation and adding the mean value.

\section{Input Selection using Genetic Algorithm}

From a modelling perspective, using only the most important variables provides a simpler and more reliable model. Even when using a good criterion for variable selection, there is no guarantee that a model created using a set of variables is optimal without testing all the possible combinations. Therefore, the input selection process must rely on heuristic criteria that can be computationally prohibitive since it involves building a model for each possible variable combination. Due to this, all the methods used in input selection for fuzzy models are suboptimal methods meaning that they cannot guarantee the optimality of the solution as they do not perform an exhaustive search. The most common methods used for input selection in ANFIS related applications are forward selection and backward selection [12], [13]. However, some studies make use of GA to find a suboptimal set of variables to build the model [14], [15].

GA is a methodology for solving optimization problems by employing a stochastic search simulating the biological evolution based on chromosomes. For the input selection problem, the chromosome can be built as a binary string that encodes the candidate variables. Equation (1) shows the fitness function used for the GA algorithm being $N$ the number of variables, $e_{c}$ and $e_{t}$ the testing and training errors respectively, $\alpha$ an influence coefficient, $\beta$ a penalty coefficient and $\gamma$ a coefficient used to increase the value of the fitness function when the constraints are not satisfied.

$$
f_{\text {fitness }}\left\{\begin{array}{cl}
\gamma\left(\operatorname{Max}_{N}-N\right) & \text { if } N>\operatorname{Max}_{N} \\
\gamma\left(\operatorname{Min}_{N}-N\right) & \text { if } N<\operatorname{Min}_{N} \\
\alpha e_{c}+(1-\alpha) e_{t}+\beta N & \text { otherwise }
\end{array}\right.
$$

\section{FDD approach with ANFIS}

An ANFIS model depends on a lot of parameters such as the number of membership functions (MF) and their shape, the number of rules, etc. The most common way of generating a FIS is by using what is called grid partitioning, based on dividing each input space using a grid to then define a membership function for each of the cells generated. Then, one rule is created for each of the possible combinations of MF. However, this method suffers what is known as curse of dimensionality when the number of inputs variables increase (being the upper limit around 5 variables). In this study, this problem is solved by using Fuzzy C-Means clustering (FCM) to group the samples in clusters, then one gaussian MF is created per cluster for each input variable and only one rule per cluster is defined.

The FDD framework is then tested by inducing some artificial faults to the dataset samples. These artificial faults are intended to decrease the chiller's coefficient of performance (COP) by increasing the energy consumed to achieve the same impulsion temperature or a decrease of this impulsion temperature without a decrease in the 
chiller's electrical consumption. In order to detect the faults, the samples are compared with those forecasted by the model. This comparison is carried out by establishing a moving window to detect samples that are 2.5 standards deviations away from the mean value predicted. These points are marked as faulty and processed following the subsequent rules:

1. Isolated faulty points are rejected considering them as false alarms.

2. Faulty samples are grouped to avoid considering them as more than one fault.

3. The fault groups are classified according to their difference with the predicted signal.

By applying this methodology, the number of false alarms is reduced drastically without sacrificing the accuracy of the model.

\section{Results and discussions}

This section demonstrates the performance of the chiller's load forecasting and the detection of faulty regions in its operation. Following the methodology described in the last section, the modelling and prediction results are first reviewed and then the fault detection is detailed in the next subsection.

\section{Energy Consumption Model}

As aforementioned, the dataset is comprised by the chiller operation related variables from the years 2017 to 2018 . Chillers are machines that are only turned on during the cooling season which covers from late May to late October. Hence, in practice, the data is reduced to more or less 12 months, Fig. 5 shows the load profile during 2017 where each peak cluster represents a week. Two different kinds of profiles can be observed: (1) Low consumption zones in the first four peaks and the last five and (2) High consumption zones where peaks can even double the consumption seen in the other category. The training dataset for the ANFIS model should contain samples corresponding to both profile types in order to accurately predict the power consumption. For this reason, the full 2017 cooling season (8648 samples) has been used to train the model assuming that the majority of the samples correspond to normal operation.
This training dataset has been split into two subsets: one for training and one for validating the model. The generated model shows a CV-RMSE of $3.56 \%$ when predicting the power consumption in the validation dataset. Other performance metrics, such as the MAE and MAPE, show values of $0.58 \mathrm{~kW}$ and $7.61 \%$ respectively. Fig. 4 shows two different days of the validation dataset used for evaluating the model performance. In general, the prediction obtained is able to resemble quite well the power consumption signal. However, there are some slight consumption changes that are not correctly modelled. This variations between the prediction and the real consumption are due to the fastest dynamics of the chiller operation. These dynamics are not included in any of the variables used as inputs thus making impossible their prediction by the ANFIS model.
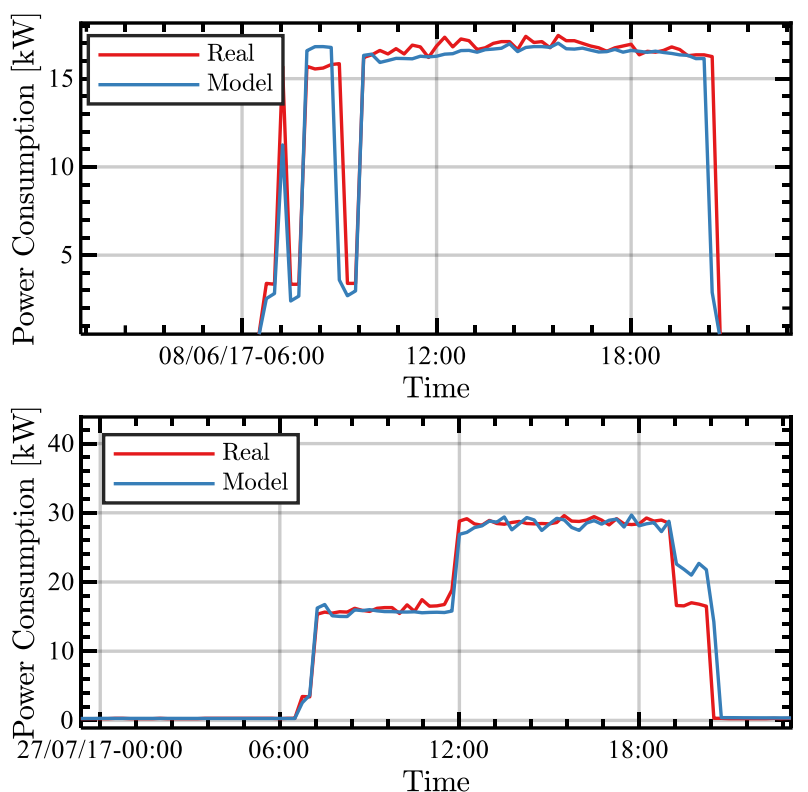

Fig. 4. Comparison between the chiller power demand signal and its prediction.

\section{B. Fault Detection Results}

In this study, the faults are introduced artificially into the power consumption profile. Several types of faults in chillers end up increasing the energy required to achieve the impulsion temperature which is able to fulfil the spaces setpoints. That is, a reduction of the COP of the chiller.

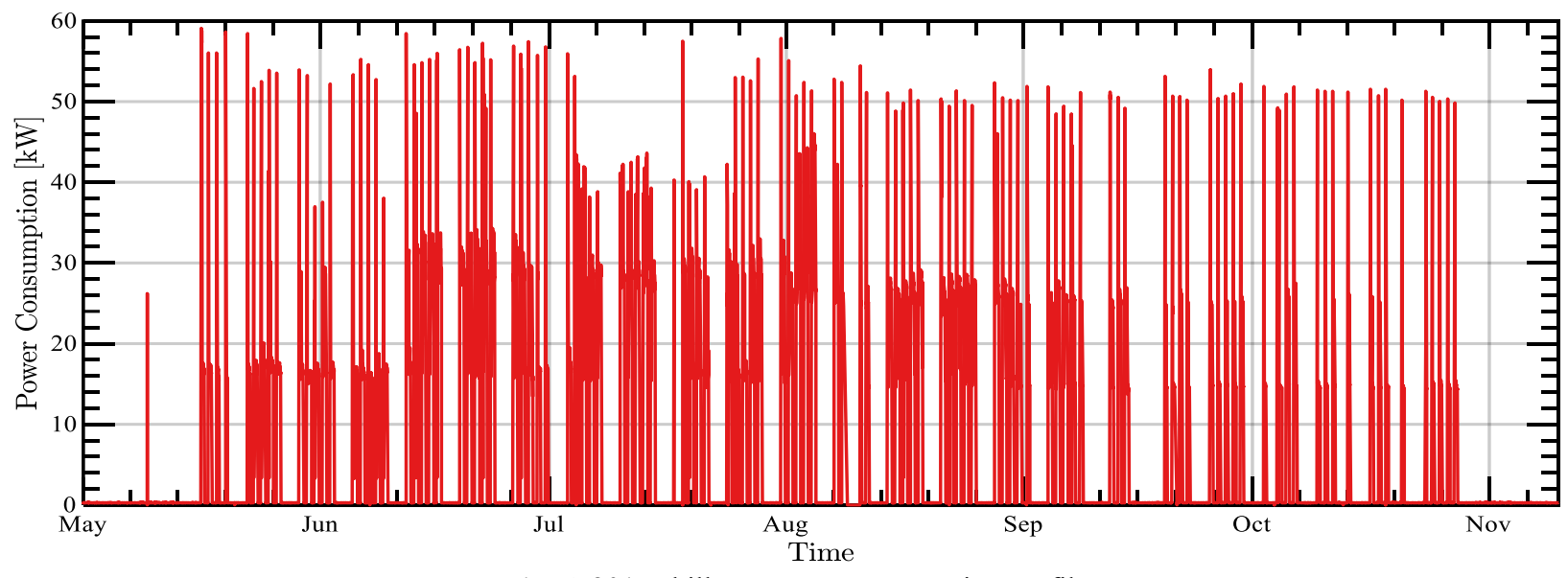

Fig. 5.2017 chiller's power consumption profile. 
Here, two different types of faults have been studied, one that produces an abrupt change on the power consumption and another that increases it in a slow manner, simulating a soft fault on one of the chiller's components. The former is relatively easy to notice using a regression-based fault detection system, so here the focus will be on detecting the second type of faults. These faults were introduced in the 2018 dataset and then the fault detection methodology proposed has been applied.

A two-day prediction where an abrupt increase of power consumption has been introduced in the first day is depicted in Fig. 6. The comparison between the predicted consumption and the faulty profile is done by calculating the difference between the real signal and the mean of the prediction using a moving window of 10 samples. The differences greater than 2.5 standard deviations are marked as fault candidate points.

\begin{tabular}{|lllll|}
\hline- Real & $\Delta$ & $5-10 \%$ & $\Delta$ & $>20 \%$ \\
\hline- Predicted & $\Delta$ & $10-20 \%$ & & \\
\hline
\end{tabular}

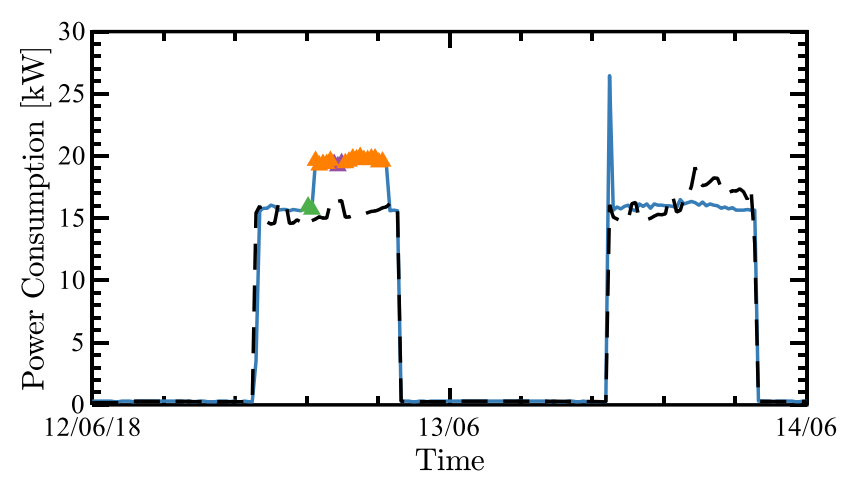

Fig. 6. Abrupt change in power consumption.

This methodology would detect the transient consumption peaks produced when the chiller is turned on as fault points. In order to avoid those false alarms, the methodology proposed include several filters that have to be bypassed by the fault candidate points. The first filter consists of neglecting isolated points. In this study, if a point is not surrounded by another faulty sample in a time span of 30 minutes it is considered to be a false alarm. After that, the faults originated by a possible error in the prediction or sensor faults are also filtered. To do so, only those fault points sets that have a time span of at least 3 hours are considered as faults. As observed in Fig. 6, this strategy is able to detect accurately abrupt increases of power consumption. However, it is also clear that this methodology also misses the opportunity to detect deviations that last less than 3 hours.

Next, the same strategy is applied to the detection of soft faults. Fig. 7 shows the result of gradually increasing power consumption for three weeks to a total increase of $15 \%$. The schema shows a fast response, as the fault is detected only one day after its introduction when the energy increment is less than $10 \%$. After its detection, continuous alarms will be raised to the maintenance personnel until the fault is corrected. It is worth mentioning that in this case, the peaks corresponding to the transient regime are also detected as faults as they are surrounded by true faulty samples.

\section{Conclusions}

This work presents a fault detection framework based on the generation of a regression model trained using a low number of faulty data samples using the ANFIS methodology.

The model is used to predict the energy consumption of the chiller under a set of circumstances to then compare the result with the one measured directly from the equipment. The prediction error is assumed to be low when the chiller operates at normal conditions, this is when there are no faults. When a fault occurs, the behaviour of the real signal deviates from the prediction. A dynamic threshold is applied to compare the difference between the measurements and the predictions. The results of the fault detection scheme are promising as they have a low rate of false alarms, high accuracy given the low error obtained in the model training and a low time delay between the error appearance and its detection.

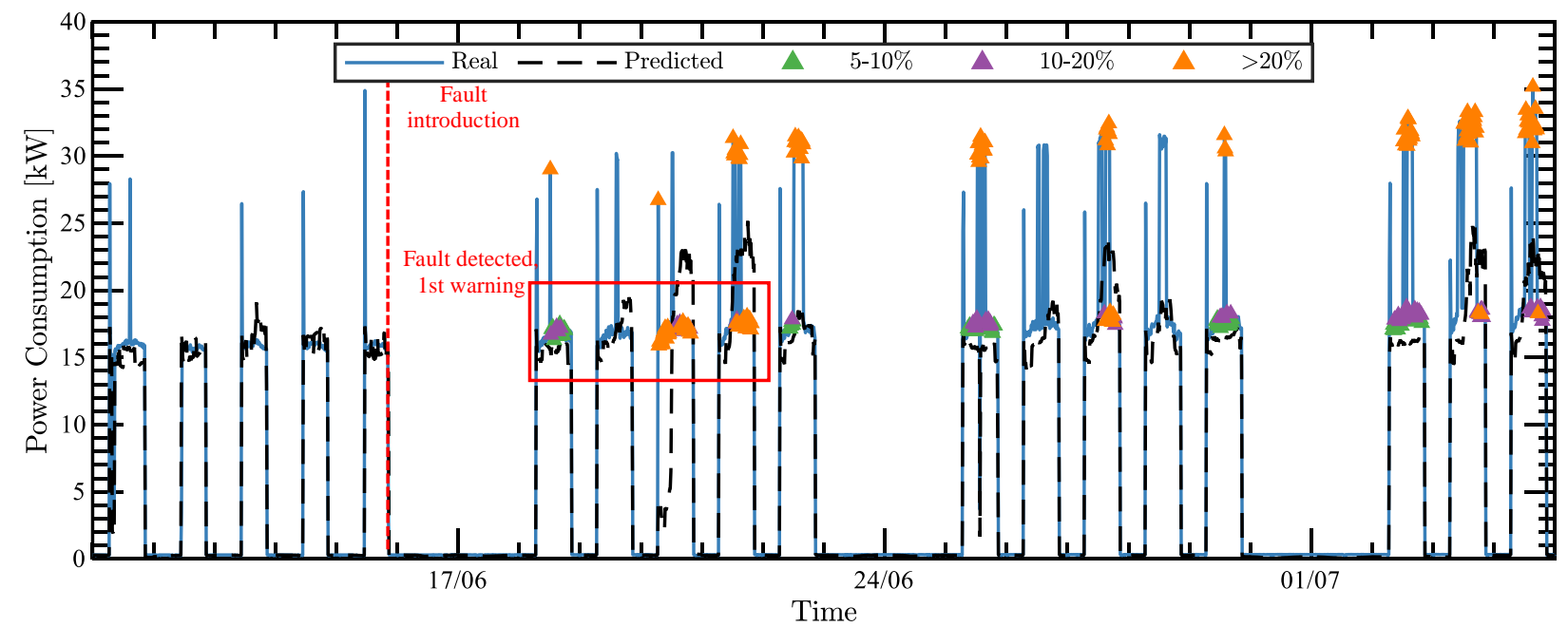

Fig. 7. Slow decrease of the chiller COP. 
The methodology proposed has two parameters that control the number of false alarms raised and the opportunities missed: Studying ways of dynamically setting these parameters can be an interesting topic of future research. The proposed fault detection is expected to reduce equipment downtime caused by major maintenance and prolong the equipment life by locating the faults in early stages before they can cause any major damage to the system. In addition, this scheme can be further combined with a classification method which, using only the data marked as a fault, could determine the type of the fault.

\section{Acknowledgement}

The authors would like to thank the support provided by the Catalan Agency for Management of University under the grant 2017 SGR 967.

\section{References}

[1] International Energy Agency (IEA), "Energy Efficiency 2018 - Analysis and outlooks to 2040," 2018.

[2] European Commission, "Directive (EU) 2018/844 of the European Parliament and of the Council of 30 May 2018 amending Directive 2010/31/EU on the energy performance of buildings and Directive 2012/27/EU on energy efficiency," in Official Journal of the European Union, vol. 61, no. June 2018, 1987, pp. 98-107.

[3] K. Yan, C. Zhong, Z. Ji, and J. Huang, "Semi-supervised learning for early detection and diagnosis of various air handling unit faults," Energy Build., vol. 181, pp. 75-83, 2018.

[4] Y. Zhao, T. Li, X. Zhang, and C. Zhang, "Artificial intelligence-based fault detection and diagnosis methods for building energy systems: Advantages, challenges and the future," Renew. Sustain. Energy Rev., vol. 109, no. February, pp. 85-101, 2019.

[5] K. Yan, L. Ma, Y. Dai, W. Shen, Z. Ji, and D. Xie, "Cost-sensitive and sequential feature selection for chiller fault detection and diagnosis," Int. J. Refrig., vol.
86, pp. 401-409, 2018.

[6] Y. Hu, G. Li, H. Chen, H. Li, and J. Liu, "Sensitivity analysis for PCA-based chiller sensor fault detection," Int. J. Refrig., vol. 63, pp. 133-143, 2016.

[7] G. Li and Y. Hu, "Improved sensor fault detection, diagnosis and estimation for screw chillers using density-based clustering and principal component analysis," Energy Build., vol. 173, pp. 502-515, 2018.

[8] J. R. Jang, “ANFIS : Adaptive-Network-Based Fuzzy Inference System," IEEE Trans. Syst. Man, Cybern., vol. 23, no. 3, 1993.

[9] Y. Ding, Q. Zhang, T. Yuan, and K. Yang, "Model input selection for building heating load prediction: A case study for an office building in Tianjin," Energy Build., vol. 159, pp. 254-270, 2018.

[10] S. Sekhar Roy, R. Roy, and V. E. Balas, "Estimating heating load in buildings using multivariate adaptive regression splines, extreme learning machine, a hybrid model of MARS and ELM," Renew. Sustain. Energy Rev., vol. 82, no. May 2017, pp. 4256-4268, 2018.

[11] X. Yu, S. Ergan, and G. Dedemen, “A data-driven approach to extract operational signatures of HVAC systems and analyze impact on electricity consumption," Appl. Energy, vol. 253, no. July, p. 113497, 2019.

[12] K. Mohammadi, S. Shamshirband, D. Petković, P. L. Yee, and Z. Mansor, "Using ANFIS for selection of more relevant parameters to predict dew point temperature," Appl. Therm. Eng., vol. 96, no. December 2018, pp. 311-319, 2016.

[13] M. L. Hadjili and V. Wertz, "Takagi-Sugeno fuzzy modeling incorporating input variables selection,' IEEE Trans. Fuzzy Syst., vol. 10, no. 6, pp. 728-742, 2002.

[14] M. Latah, "Anfis Approach With Genetic Feature Selection for Prediction of Students' Academic Performance in Distance Education Environment," Int. J. Eng. Appl. Sci. Technol., vol. 1, no. 8, pp. 24552143, 2016.

[15] K. Kampouropoulos, "Multi-Objective Optimization of an Energy Hub using Artificial Intelligence," Universitat Politècnica de Catalunya, 2018. 